\title{
Sociodemographic and Regional Disparities in the Prevalence of Self-Reported History of Coronary Heart Disease among Persons 18 Years and Over in the United States
}

\author{
Frantz D. Soiro, BA ${ }^{1}$; Mechelle D. Claridy, MPH ${ }^{1}$; Stephanie Miles-Richardson, DVM, PhD $^{1}$; \\ and Gemechu B. Gerbi, MSc, PhD*1 \\ ${ }^{1}$ Morehouse School of Medicine,720 Westview Dr. SW, NCPC Suite 346, Atlanta, GA 30310-1495 USA \\ *Corresponding Author: Gemechu B. Gerbi. MSc. PhD, Morehouse School of Medicine, 720 Westview \\ Dr.SW, NCPC Suite 346, Atlanta, GA 30310-1495 USA, Email: ggerbi@msm.edu
}

\begin{abstract}
Background: Heart disease accounts for about 1 in 4 deaths with coronary heart disease (CHD) being responsible for over 370,000 deaths per year. The objective of this study was to assess factors associated with the prevalence of CHD among persons aged $\geq 18$ years in the United States (US).

Methods: Data were analyzed from the 2018 Behavioral Risk Factor Surveillance System, an ongoing, state-based, random-digit-dialed telephone survey of non-institutionalized adults aged $\geq 18$ years residing in the US. A multivariable logistic regression analysis was conducted to estimate adjusted odds ratios (AORs) and $95 \%$ confidence intervals (95\% CIs) for factors associated with CHD among persons aged $\geq 18$ years in the U.S. Analyses were conducted using SAS version 9.4.

Results: Of the 433,763 participants, 26,114 (6\%) reported history of CHD while 407, 649 (94\%) did not report history of CHD. Factors associated with higher odds of reporting a history of CHD included: being male $(A O R=2.10 ; 95 \% C I=2.01,2.13)$; being multiracial $(A O R=1.33 ; 95 \% C I=1.19,1.47)$; having an annual household income of $\$ 15,000$ or less $(A O R=2.23 ; 95 \% C I=2.12-2.34)$, earning $\$ 15,000$ to $\$ 24,999$ per year $(A O R=1.73 ; 95 \% C I=1.66-1.81)$, earning $\$ 25,000$ to $\$ 34,999$ per year $(A O R=1.36 ; 95 \% C I=$ 1.29-1.43), and earning $\$ 35,000$ to $\$ 49,999$ per year $(A O R=1.24 ; 95 \% C I=1.18-1.30)$; residing in the South $(A O R=1.42 ; 95 \% C I=1.36,1.48)$, residing in the Midwest (AOR: 1.16; 95\% CI, 1.11-1.22), and residing in the Northeast (AOR: 1.11; 95\% CI, 1.05-1.16).Persons who did not participate in any physical activities or exercises $(A O R=1.47 ; 95 \% C I=1.42-1.52)$ were also more likely to report a history of $C H D$.

Conclusion: The prevalence of self-reported history of CHD diagnosis differs by sociodemographic factors, exercise, and region of residence. Developing targeted interventions, especially for high-risk populations, is needed to reduce the risk for CHD among adults in the US.
\end{abstract}

Keywords: Self-reported CHD, persons aged $\geq 18$ years, BRFSS, United States

\section{INTRODUCTION}

Coronary heart disease (CHD) is a public health concern that has caused many deaths and disabilities worldwide. According to the Centers for Disease Control and Prevention (CDC), CHD is defined as a plaque build-up in the walls of the arteries that provide blood to the heart. This plaque build-up is made of cholesterol deposits that create a partial or total blockage of blood flow over time[1].Terminology such as coronary heart disease, coronary artery disease, cardiomyopathy, and heart failure are often used interchangeably. In the United States (US), CHD remains the leading cause of death among men and women across most racial and ethnic groups[1, 2]. In 2017, CHD was responsible for over 370,000 deaths and approximately 18.2 million people over the age of 20 reported having CHD $[1,3]$.The overall mortality rates of CHD have declined over the years but there remains a heart disease burden in the US among different populations and subgroups[3].The burden of heart disease has cost the nation approximately $\$ 214$ billion per year of the $90 \%$ health care expenditures ( $\sim 3.8$ trillion) which represents chronic and mental health conditions. The treatment of CHD typically involves lifestyle changes such as quitting smoking, engaging in routine exercise, and eating healthy foods. Also, depending on the severity of one's 
health condition, drug intervention (i.e., cholesterol-modifying medication) or surgical procedures (i.e., coronary artery bypass surgery) may be recommended.

There are well-documented disparities in the treatment and survival of CHD. However, there are few research studies that have identified health inequities across geographic areas[49].Similarly, there are limited studies which examine heart disease on a national level. Specifically, factors such as sociodemographic and socioeconomic factors, physical activity, and region of residence have not been studied extensively. Therefore, a study on the association between gender, age, race/ethnicity, level of income, level of education, regiaon of residence, physical activity, and CHD utilizing a nationally representative sample can add to the current body of literature surrounding heart disease burden topics in the US.

An assessment of sociodemographic and regional disparities at the national level will help provide states and local communities with documentation of specific health disparities. With statistical data, public health practitioners can tailor their prevention approaches based on the epidemiologic trends of coronary heart disease. The objective of this study is to assess the factors associated with the prevalence of CHD among adults aged $\geq 18$ years in the US.

\section{METHODS}

\subsection{Data Source and Study Sample}

Data were analyzed from the 2018 Behavioral Risk Factor Surveillance System (BRFSS), an ongoing, state-based, random-digit-dialed telephone survey of non-institutionalized adults aged $\geq 18$ years residing in the US. The BRFSS is a collaborative project between all 50 states in the US, the District of Columbia, three US territories (Puerto Rico, Guam, and the US Virgin Islands) and the Centers for Disease Control and Prevention (CDC). It collects statespecific data on health risk behaviors, chronic diseases and conditions, access to health care, and use of preventive health services related to the leading causes of death and disability in the US [10]. A clustering sample design was used to account for differences in the probability of selection and nonresponse to accurately derive US and state-based population estimates[10]. Annual surveys consist of a core set of questions about various health topics such as healthrelated risk behaviors, chronic health conditions, and the use of preventive services. The CHD question used for this study was taken from the core questions. The BRFSS data are publicly available via the CDC website: www.cdc.gov/ brfss/annual_data/annual_2018.html and are used for health policy development and advocacy at both the national and state levels.

\section{MeASURES}

All measures in the study were based on the self-reported data obtained from the 2018 BRFSS.

\subsection{Dependent Variable (CHD)}

The prevalence of CHD was determined if the participants answered "yes" to the question: "(Ever told) you had angina or coronary heart disease?" Possible responses included "yes", "no", "don't know/not sure", or "refused". Records with "unknown or "refused" responses or missing data were excluded from the analysis.

\subsection{Covariates and Independent Variables}

Participants' gender, race/ethnicity, age, level of education, level of income, region of residence and physical activity status were assessed from the 2018 BRFSS. To examine the role of region of residence on CHD, individual's region of residency was categorized as Black Belt (Virginia, North Carolina, South Carolina, Georgia, Florida, Alabama, Mississippi, Tennessee, Louisiana, Arkansas, Texas), or the remainder of the Southern US (Delaware, District of Columbia, Maryland, West Virginia, Kentucky, Oklahoma), Midwest, Northeast, and West. Self-reported exercise was determined if the participants answered "Yes" to the Question: "During the past month, other than your regular job, did you participate in any physical activities or exercises such as running, calisthenics, golf, gardening, or walking for exercise? Possible responses include "yes," "no," "don't know/not sure," or "refused." Records with "unknown" or "refused" responses or missing data were excluded from the analysis.

\subsection{Statistical Analysis}

Descriptive statistics were used to compute frequencies. A bivariate analysis, using the chisquare test statistic $(\chi 2)$, was used to conduct an initial assessment of the factors independently associated with self-reported history of CHD. All variables that had achieved $p \leq 0.05$ in the bivariate analysis were included in the multivariable logistic regression model to obtain the adjusted odds ratios (AORs) and 95\% 
Predictors of Complementary and Alternative Medicine Use by Yoruba and Hausa People in Urban Slum Areas of Ibadan, Nigeria

confidence intervals (95\% CIs). A 2-sided $\mathrm{p}$ value of $\leq 0.05$ was considered to indicate statistical significance. All statistical analyses were performed using SAS version 9.4[11].

\section{RESULTS}

Table 1 shows a summary of the select characteristics of the respondents by number and percentages in relation to their gender, age, race/ethnicity, level of education, level of income, region of residence, and exercise status. Of the 433,763 participants who responded to

Table1. Number and percentage $e^{\dagger}$ of respondents who reported having history of coronary heart disease by select characteristics: 2018 BRFSS, United States

\begin{tabular}{|c|c|c|c|c|}
\hline \multirow[t]{2}{*}{ Select Characteristics } & \multicolumn{3}{|c|}{$\begin{array}{l}\text { Self-reported history of coronary heart disease } \\
(\mathrm{N}=433,763)\end{array}$} & \multirow[b]{2}{*}{ p-value } \\
\hline & Yes & No & Total & \\
\hline & n (\%) & n (\%) & n (\%) & \\
\hline Overall & $26114(6)$ & $407649(94)$ & $433763(100)$ & \\
\hline Gender & & & & $<0.0001$ \\
\hline Male & $14659(5)$ & $181017(95)$ & $195676(100)$ & \\
\hline Female & $11370(8)$ & $225690(92)$ & $237060(100)$ & \\
\hline Race/Ethnicity & & & & $<0.0001$ \\
\hline White, Non-Hispanic & $20757(6)$ & $301427(94)$ & $322184(100)$ & \\
\hline Black, Non-Hispanic & $1846(5)$ & $33789(95)$ & $35635(100)$ & \\
\hline Hispanic & $1304(4)$ & $35402(96)$ & $36706(100)$ & \\
\hline Multiracial & $572(7)$ & $7848(93)$ & $8420(100)$ & \\
\hline Other & $1102(5)$ & $21470(95)$ & $22572(100)$ & \\
\hline Age Group & & & & $<0.0001$ \\
\hline $18-24$ & $87(1)$ & $25860(99)$ & $25947(100)$ & \\
\hline $25-34$ & $257(1)$ & 46249 (99) & $46506(100)$ & \\
\hline $35-44$ & $678(1)$ & $51633(99)$ & $52311(100)$ & \\
\hline $45-54$ & $2048(3)$ & $65353(97)$ & $67401(100)$ & \\
\hline $55-64$ & $5472(6)$ & $84471(94)$ & $89943(100)$ & \\
\hline$\geq 65$ & $17572(12)$ & $134083(88)$ & $151655(100)$ & \\
\hline Level of Education & & & & $<0.0001$ \\
\hline Did not graduate High School & $47(7)$ & $60793)$ & $654(100)$ & \\
\hline Elementary & $937(9)$ & $9284(91)$ & $10221(100)$ & \\
\hline Some high school & $9266(7)$ & $130852(93)$ & $140118(100)$ & \\
\hline High school graduate & $8040(7)$ & $109736(93)$ & $117776(100)$ & \\
\hline College graduate & $7752(5)$ & $155732(95)$ & $163484(100)$ & \\
\hline Level of Income & & & & $<0.0001$ \\
\hline Less than $\$ 15,000$ & $3361(10)$ & $30843(90)$ & $34204(100)$ & \\
\hline$\$ 15,000-\$ 24,999$ & $4828(8)$ & $52593(92)$ & $57421(100)$ & \\
\hline$\$ 25,000-\$ 34,999$ & $2617(7)$ & $34875(93)$ & $37492(100)$ & \\
\hline$\$ 35,000-\$ 49,999$ & $3076(6)$ & $46183(94)$ & $49259(100)$ & \\
\hline More than $\$ 50,000$ & $7716(4)$ & $172762(96)$ & $180478(100)$ & \\
\hline Region of Residence & & & & $<0.0001$ \\
\hline South & $8440(7)$ & $109565(93)$ & $118005(100)$ & \\
\hline Midwest & $6609(6)$ & $104053(94)$ & $110662(100)$ & \\
\hline Northeast & $5157(6)$ & $85378(94)$ & $90535(100)$ & \\
\hline West & $4375(5)$ & $87446(95)$ & $91821(100)$ & \\
\hline Exercise & & & & $<0.0001$ \\
\hline Yes & $16066(5)$ & $308358(95)$ & $324424(100)$ & \\
\hline No & $9990(9)$ & $98733(91)$ & $108723(100)$ & \\
\hline
\end{tabular}

Note: *Frequencies may vary due to missing values

Table 2 shows the results of multivariable logistic regression adjusted for

sociodemographic characteristics (gender, age, race/ethnicity, level of education, level of the question: "(Ever told) you had angina or coronary heart disease?", 26,114 (6\%) reported having had CHD, and 407,649 (94\%) reported not having had CHD. The majority of respondents who reported having had CHD were females (44\%), White $(79 \%), \geq 65$ years of age $(67 \%)$, had Elementary education $(3 \%)$, had annual household income level of less than $\$ 15,000(13 \%)$, resided in the South (32\%), and did not participate in any physical activities or exercises $(38 \%)$. 
Predictors of Complementary and Alternative Medicine Use by Yoruba and Hausa People in Urban Slum Areas of Ibadan, Nigeria

income, region of residence) and exercise status. In comparison to female respondents, males were significantly more likely to report history of $\mathrm{CHD}$ (AOR=2.10; 95\% CI, 2.01-2.13). Compared with participants with an annual household income of $\$ 50,000$ or more, those with an annual household income of $\$ 15,000$ or less had higher odds of self-reporting a history of $\mathrm{CHD}(\mathrm{AOR})=2.23$; 95\% CI, 2.12-2.34); as those with an annual household income of $\$ 15,000$ to $\$ 24,999$ (AOR $=1.73 ; 95 \% \mathrm{CI}, 1.66$ $1.81), \$ 25,000$ to $\$ 34,999$ ( $\mathrm{AOR}=1.36$; $95 \% \mathrm{CI}$, 1.29-1.43) and $\$ 35,000$ to $\$ 49,999(\mathrm{AOR}=1.24$; 95\% CI, 1.18-1.30). Compared with participants with a college graduate education, those with an

Table2. Adjusted odds ratios and 95\% confidence intervals for factors associated with self-reported history of coronary heart disease by select characteristics: 2018 BRFSS, United States

\begin{tabular}{|l|l|l|}
\hline Select Characteristics & \multicolumn{2}{l|}{$\begin{array}{l}\text { Self-reported history of Coronary Heart Disease } \\
\text { Yes }\end{array}$} \\
\hline & AOR & $\mathbf{9 5 \%}$ CI \\
\hline Gender & & \\
\hline Male & 2.10 & $2.01-2.13$ \\
\hline Female & Ref & \\
\hline Race/Ethnicity & & \\
\hline White, Non-Hispanic & Ref & \\
\hline Black, Non-Hispanic & 0.75 & $0.70-0.80$ \\
\hline Hispanic & 0.72 & $0.66-0.78$ \\
\hline Multiracial & 1.33 & $1.19-1.47$ \\
\hline Other & 1.01 & $0.93-1.09$ \\
\hline Age Group & & \\
\hline 18 to 24 & Ref & \\
\hline 25 to 34 & 1.70 & $1.27-2.31$ \\
\hline 35 to 44 & 4.45 & $3.41-5.91$ \\
\hline 45 to 54 & 10.69 & $8.28-14.10$ \\
\hline 55 to 64 & 21.58 & $16.78-28.37$ \\
\hline 65 or older & 44.75 & $38.83-58.80$ \\
\hline Level of Education & & \\
\hline Did not graduate High School & 1.03 & $0.64-1.57$ \\
\hline Elementary & 1.27 & $1.15-1.40$ \\
\hline Some high school & 1.14 & $1.09-1.19$ \\
\hline High school graduate & 1.25 & $1.20-1.30$ \\
\hline College graduate & Ref & \\
\hline Annual Household Income & & \\
\hline Less than $\$ 15,000$ & 2.23 & $2.12-2.34$ \\
\hline \$15,000-\$24,999 & 1.73 & $1.66-1.81$ \\
\hline \$25,000-\$34,999 & 1.36 & $1.29-1.43$ \\
\hline \$35,000-\$49,999 & 1.24 & $1.18-1.30$ \\
\hline More than $\$ 50,000$ & Ref & \\
\hline Region of Residence & & $1.36-1.48$ \\
\hline South & 1.42 & $1.11-1.22$ \\
\hline Midwest & 1.16 & \\
\hline Northeast & 1.11 & \\
\hline West & Ref & $1.42-1.52$ \\
\hline Exercise & & \\
\hline Yes & Ref & \\
\hline No & 1.47 & \\
\hline & & \\
\hline & & \\
\hline & & \\
\hline & & \\
\hline & & \\
\hline
\end{tabular}

Note: $\mathrm{AOR}=$ adjusted odds ratio; $\mathrm{CI}=$ confidence interval education level of a high school graduate $(\mathrm{AOR}=1.25 ; 95 \% \mathrm{CI}, 1.20-1.30)$, some high school $(\mathrm{AOR}=1.14 ; 95 \% \mathrm{CI}, 1.09-1.19)$, elementary $(\mathrm{AOR}=1.27$; 95\% CI, 1.15-1.40), and those that did not graduate high school $(\mathrm{AOR}=1.03 ; 95 \% \mathrm{CI}, 0.64-1.57)$ were more likely to self-report a history of CHD. Respondents residing in the South ( $\mathrm{AOR}=1.42$; CI, 1.11-1.22), Northeast $(A O R=1.11 ; 95 \%$ CI, 1.05-1.16); and those who did not participate in any physical activities or exercises $(\mathrm{AOR}=1.47$; 95\% CI, 1.42-1.52) were also more likely to report history of CHD. 95\% CI, 1.36-1.48), Midwest (AOR $=1.16 ; 95 \%$ 


\section{DisCUSSION}

Our findings show that six percent of the 2018 BRFSS survey participants who responded to the CHD core question reported having had a history of CHD. The most reasonable explanation of this finding is a person's perception of their health status. A previous study observed fewer participants with selfreported history of CHD from the same database indicating an increase trend in prevalence rate [12].Our study is among a few studies to provide an assessment of the prevalence of coronary heart disease and its correlation to geographic and sociodemographic disparities using a nationally representative populationbased sample in the US.

Our findings show that male respondents were more likely to report a history of coronary heart disease than were female respondents. Interestingly, this data provides a mixed picture with the literature $[13,14]$ and is plausibly due to the inadequate processes of monitoring heart disease prevalence across the US. Particularly, studies have reported that men under 55 years are approximately 4 times more likely to have a serious manifestation of CHD such as myocardial infarction compared to women[13]. Additionally, Graham, G. (2015) reports that men are more likely to be hospitalized for heart disease and myocardial infarction while women are more likely admitted for congestive heart failure[14].However, researchers such as Shufelt et al. (2019) points to the dramatic changes of hormone-mediated physiology and associate women being more at risk of cardiovascular comorbidities[15].

The current study found that racial/ethnic differences in the prevalence of self-reported CHD was more likely in those who identified as multiracial. Studies have shown that races other than White (Non-Hispanic) adults have poorer health conditions of CHD. A study that assessed cardiovascular disease disparities in the US reported that African Americans have among the highest rates of hypertension in the world. Subsequently, they and Mexican Americans present lower levels of blood pressure control compared to their white counterparts even after adjusting for modifiable health behaviors[14]. Findings in this study suggested that African Americans have a 30\% increased likelihood of dying from heart disease, Asian American/ Pacific Islanders experience early onset and higher percentages of coronary heart disease than in any other ethnic group, and Non-White
Hispanics such as Puerto Rican Americans have the highest hypertension related death rates among all Hispanic sub groups [14]. These findings are attributable to limited access to care and access to needed resources, chronic stress, lack of heart disease management infrastructure, and systemic racism. This builds on previous research urging the need for improved outcomes among various populations across the nation. This drives the need to create tailored "culturally sensitive" interventions for health care professionals. Without understanding the social, cultural, and linguistic needs of diverse populations, minority and underserved communities will not realize a decline in heart disease mortality. To mitigate these disparities, cultural competency should be the focus, which will provide an understanding of diverse values, beliefs, and behaviors among those at-risk[14].

Our findings show that respondents who had an annual household income less than $\$ 50,000$ were more likely to report having had a history of CHD. Previous studies have also shown that household income was strongly and independently associated with CHD [16]. For example, $10.6 \%$ of those who had a household income of $\$ 29,999$ per year or less had CHD compared with $2.7 \%$ of those who made $\$ 80,000$ or more per year[17]. Lemstra and colleagues suggest that low income has a more important association with CHD than conventional risk factors such as smoking and physical inactivity do, suggesting that a reordering of risk factors is required [17]. Another study found that $9.2 \%$ of low-income people had CHD compared with $2.9 \%$ of highincome people [16]. This study also demonstrated that the top income earners had a high blood pressure prevalence of $7.3 \%$ while the lowest earners had a prevalence of $15.4 \%$ [16]. A study by Gerber and colleagues (2008) found that those who received an increase in median income of approximately $\$ 10,000$ were $10 \%$ less at-risk of dying from CHD [18].Also, residents in low-income communities tend to not adhere to medications or undergo recommended interventions given the cost association[19].The most possible explanation for the statistically significant association observed between household income and CHD is that lower-income individuals have more stress, including insecurity in income, housing, food, safety, while also having fewer resources to address these challenges[20]. The mismatch between demands that individuals live with, 
coupled with the reduced capacity to cope effectively, results in increased distress and subsequently, risky behaviors, which leads to CHD.

Our findings show that those who have less than a college graduate level education was more likely to report having a history of CHD. Previous studies have shown us that there is a strong correlation of education and health. For example, $56.6 \%$ of CHD risks in people with low education were strongly associated with behavioral and biological risk factors such as smoking $(27.3 \%)$, obesity (10.2\%), physical inactivity (6.3\%), and hypertension (5.3\%)[19]. Schultz and colleagues (2018) suggest that the gap in education and health potentially lies with health literacy, proving that people with poor health literacy are more likely noncompliant with medication, therefore at-risk of underlying health conditions and have an increased experience of cardiovascular disease mortality [19].Another study found that education attainment independent of cognition had a strong protective effect against CHD. This study showed that high education attainers had a healthier lifestyle, safer working conditions at their workplace, and better access to health care. Therefore, these individuals are less likely to engage in harmful behaviors such as smoking and alcohol intake due to informed knowledge of the negative effects of such behaviors [21]. A study found that men and women with less than high school education had $73.7 \%$ and $48.2 \%$ higher 10-year CHD risk compared to those with at least a college degree, respectively[22]. This study suggests that this level of significance has a strong causal association with the importance of reading comprehension among men and women. Also, with an increase in educational attainment simultaneously increases ones perceived control. A prospective study of 3,888 participants showed associations between education and incident CHD were reduced by $27 \%$ after adjusting for sense of control[21].With education attainment, people have the capability to make informed decisions about what influences their behavior which affects their exposure to CHD.

Our findings also show that respondents who reside in the Southern region of the US were more likely to report a history of CHD compared with those who reside in the Midwest, Northeast, or West. Findings from this analysis were aligned with previous research on geographic variation in the prevalence of cardiovascular disease and historical reference of the "South" being a "stroke and heart disease belt" in the United States [7, 8, 23]. All 4 US regions resulted in being at risk of reporting a history of CHD, however, those who reside in the Midwest, Northeast, and West tend to bear slightly less of the disease burden than the South. For example, a study showed that the southeast region of the US had a 10\%-point increase in obesity and diabetes prevalence increased the percentage points of cardiovascular diseases (32.2\% and 57.7\%)[8]. These same underlying health conditions are more prevalent in southern states compared to other regions across the US [24]. Casper and colleagues (2016) provide a temporal change data in heart disease percentages across the US showing a dramatic drop from $48 \%$ to $4 \%$ of northern counties, $17 \%$ to $6 \%$ of midwestern counties, while an opposite trend of $24 \%$ to $38 \%$ in southern counties[24]. Similarly, in a previously mentioned study by Schultz and colleagues, provided a geomapping figure that showed regional clustering of heart disease hotspots that highlighted the Southern region in great need of aggressive community-based interventions [19]. Our study results, in comparison to previous studies, suggest a multilevel and multi-sectoral intervention with a comprehensive focus on social determinants of health and a coordinated effort in community engagement to heart disease management. Physical activity campaigns, smoking-cessation programs, access to health care services, and policy interventions that are tailored to residents who reside in the southern region of the US are vital. Our findings suggest that more research is needed to increase our understanding of geographical disparities in the prevalence of CHD.

The current study also found that those who did not participate in physical activity were more likely to have a history of CHD. Our results were aligned with findings from previous research $[25,26]$ and highlights the significance of varying exercise intensity levels to CHD diagnosis [27]. For example, in a Harvard Alumni Study 10, 269 sedentary individuals who increased the amount of moderate to vigorous physical activity had a $41 \%$ lower risk of CHD compared to those who remained sedentary [28]. Another study found that a high level of leisure-time physical activity was associated with $16 \%$ and $23 \%$ reductions in 
CHD risk for men and women, respectively, compared to those with low levels of physical activity[29]. This study also demonstrated that specific physical activities benefit women (daily walking, cycling) compared to men (occupational activities). Based on our findings and previous studies, exercise or physical activity reduces CHD risk factors like diabetes mellitus or obesity and improves psychological factors that impact the heart such as stress. Adherence and compliance to exercise training yields significant gains in one's health outcomes. While basic knowledge of exercise is known to protect people from $\mathrm{CHD}$, a further exploration on exercise and quality of life is needed. Access to healthpromoting physical or built environments in disadvantaged areas impacts a populations' physical health and in turn creates an increased exposure to other preventable or modifiable risk factors to CHD [8, 30].

\subsection{Study Limitations}

Based on the research findings, limitations were considered. First, BRFSS is a telephone-based survey and is administered to civilian, noninstitutionalized adults. Hence, the BRFSS excludes people without telephone service, those on military bases, and individuals in institutions. For this reason, generalizability to the entire U.S population is limited. Second, the phone survey responses from BRFSS may be subject to recall or reporting bias. Third, as to an individual's time of diagnosis, the BRFSS question asks, "(Ever told) you had angina or coronary heart disease?" Since responses include "yes", "no", "don't know/not sure", and "refused", we cannot determine if respondents are being treated for their condition which can speak to their perception of their health status.

\section{CONCLUSiON}

The prevalence of self-reported history of CHD diagnosis differs by sociodemographic factors, exercise, and region of residence. Developing targeted interventions, especially for high-risk populations, is needed to reduce the risk for CHD among adults in the US.

\section{Public Health Implications and RECOMMENDATIONS}

These findings help provide insight to future research that aims to reduce geographic and sociodemographic disparities in the prevalence of CHD. Public health interventions and strategies should be tailored to populations most at-risk. For example, policies should be implemented to assure social and physical environments that are safe and accessible, and which promote physical activity in minority and underserved communities. Second, implementing the community navigation model in geographic areas that are highly populated with at-risk populations can help mitigate hospital readmission rates by aligning relevant resources to help treat their heart conditions. Third, create workshops and training for clinicians and other healthcare professionals to learn cultural sensitivity. This will allow for a comprehensive understanding to a patient's health condition. Lastly, investigating our regional surveillance strategies to disease burden patterns can help limit the differential rates of mortality and morbidity.

\section{REFERENCES}

[1] Prevention, C.f.D.C.a., Hear Disease Facts. 2019, National Center for Chronic Disease Prevention and Health Promotion, Division of Heart Disease and Stroke Prevention.

[2] Michimi, A., Modeling coronary heart disease prevalence in regional and sociodemographic contexts. Health \& Place, 2010. 16(1): p. 147-155.

[3] Sanchis-Gomar, F., et al., Epidemiology of coronary heart disease and acute coronary syndrome. Ann Transl Med, 2016. 4(13): p. 256.

[4] Barnett, E., et al., Metropolitan and nonmetropolitan trends in coronary heart disease mortality within Appalachia, 1980-1997. Ann Epidemiol, 2000. 10(6): p. 370-9.

[5] Gillum, R.F., et al., Racial and geographic variation in coronary heart disease mortality trends. BMC Public Health, 2012. 12: p. 410.

[6] Kulshreshtha, A., et al., Urban-rural differences in coronary heart disease mortality in the United States: 1999-2009. Public Health Rep, 2014. 129(1): p. 19-29.

[7] Pickle LW, M.M., Jones GK, White AA, Atlas of United States Mortality. 1996. DHHS Publication (National Center for Health Statistics, Centers for Disease Control and Prevention.).

[8] Singh, G.K., et al., Widening Geographical Disparities in Cardiovascular Disease Mortality in the United States, 1969-2011. Int J MCH AIDS, 2015. 3(2): p. 134-49.

[9] Vaughan, A.S., M.R. Kramer, and M. Casper, Geographic disparities in declining rates of heart disease mortality in the southern United States, 1973-2010. Prev Chronic Dis, 2014. 11: p. E185.

[10] Prevention, C.f.D.C.a., 2018 Behavioral Risk Factor Surveillance System Questionnaire. 2018.

[11] SAS Institute, C., NC, USA. 
[12] Wardoku, R., et al., Association between physical inactivity and health-related quality of life in adults with coronary heart disease. Maturitas, 2019. 128: p. 36-42.

[13] Gheisari, F., et al., The Role of Gender in the Importance of Risk Factors for Coronary Artery Disease. Cardiol Res Pract, 2020. 2020: p. 6527820 .

[14] Graham, G., Disparities in cardiovascular disease risk in the United States. Current cardiology reviews, 2015. 11(3): p. 238-245.

[15] Shufelt, C.L., et al., Sex-Specific Physiology and Cardiovascular Disease. Advances in experimental medicine and biology, 2018. 1065: p. 433-454.

[16] Lee, D.S., et al., Trends in risk factors for cardiovascular disease in Canada: temporal, socio-demographic and geographic factors. Cmaj, 2009. 181(3-4): p. E55-66.

[17] Lemstra, M., M. Rogers, and J. Moraros, Income and heart disease: Neglected risk factor. Can Fam Physician, 2015. 61(8): p. 698-704.

[18] Gerber, Y., et al., Neighborhood income and individual education: effect on survival after myocardial infarction. Mayo Clin Proc, 2008. 83(6): p. 663-9.

[19] Schultz, W.M., et al., Socioeconomic Status and Cardiovascular Outcomes: Challenges and Interventions. Circulation, 2018. 137(20): p. 2166-2178.

[20] Eaton WW, M., C, A_Handbook for the Study of Mental Health Social Contexts Theories and Systems 2nd edition.pdf. Cambridge University Press, 1999: p. 259-83.

[21] Gill, D., et al., Education protects against coronary heart disease and stroke independently of cognitive function: evidence from Mendelian randomization. Int J Epidemiol, 2019. 48(5): p. 1468-1477.

[22] Loucks, E.B., et al., Education and coronary heart disease risk: potential mechanisms such as literacy, perceived constraints, and depressive symptoms. Health Educ Behav, 2015. 42(3): p. 370-9.

[23] Feinleib, M., et al., Time trends, cohort effects, and geographic patterns in stroke mortality-United States. Ann Epidemiol, 1993. 3(5): p. 458-65.
[24] Casper, M., et al., Changes in the Geographic Patterns of Heart Disease Mortality in the United States: 1973 to 2010. Circulation, 2016. 133(12): p. 1171-80.

[25] Lavie, C.J., et al., Exercise and the cardiovascular system: clinical science and cardiovascular outcomes. Circ Res, 2015. 117 (2): p. 207-19.

[26] Swift, D.L., et al., Physical activity, cardiorespiratory fitness, and exercise training in primary and secondary coronary prevention. Circ J, 2013. 77(2): p. 281-92.

[27] Stewart, R.A.H., et al., Physical Activity and Mortality in Patients With Stable Coronary Heart Disease. J Am Coll Cardiol, 2017. 70(14): p. 1689-1700.

[28] Paffenbarger, R.S., Jr., et al., The association of changes in physical-activity level and other lifestyle characteristics with mortality among men. N Engl J Med, 1993. 328(8): p. 538-45.

[29] Hu, G., et al., Occupational, commuting and leisure-time physical activity in relation to coronary heart disease among middle-aged Finnish men and women. Atherosclerosis, 2007. 194(2): p. 490-7.

[30] Organization, W.H., Cardiovascular Diseases Key Facts. 2017, World Health Organization: Online.

[31] Bhatnagar, A., Environmental Determinants of Cardiovascular Disease. Circulation research, 2017. 121(2): p. 162-180.

[32] Fryar, C.D., T.-C. Chen, and X. Li, Prevalence of uncontrolled risk factors for cardiovascular disease: United States, 1999-2010. NCHS data brief, 2012(103): p. 1-8.

[33] Leigh, J.A., M. Alvarez, and C.J. Rodriguez, Ethnic Minorities and Coronary Heart Disease: an Update and Future Directions. Current atherosclerosis reports, 2016. 18(2): p. 9-9.

[34] Zeki Al Hazzouri, A., et al., Sustained Economic Hardship and Cognitive Function: The Coronary Artery Risk Development in Young Adults Study. Am J Prev Med, 2017. 52(1): p. 1-9.

[35] Services, U.D.o.H.a.H., Healthy People 2020.

Citation: Gemechu B. Gerbi. MSc. PhD, et.al. Sociodemographic and Regional Disparities in the Prevalence of Self-Reported History of Coronary Heart Disease among Persons 18 Years and Over in the United States. ARC Journal of Public Health and Community Medicine. 2021; 6(1):33-40. Doi:doi.org/10.20431/24560596.0601005

Copyright: (C) 2021 Authors. This is an open-access article distributed under the terms of the Creative Commons Attribution License, which permits unrestricted use, distribution, and reproduction in any medium, provided the original author and source are credited. 